\title{
\begin{tabular}{l|l} 
Mitraries & DSpace@MIT
\end{tabular}
}

\author{
MIT Open Access Articles
}

\section{A spin on cavity formation during water entry of hydrophobic and hydrophilic spheres}

The MIT Faculty has made this article openly available. Please share how this access benefits you. Your story matters.

Citation: Truscott, Tadd T., and Alexandra H. Techet. "A spin on cavity formation during water entry of hydrophobic and hydrophilic spheres." Physics of Fluids 21 (2009): 121703. (C) 2009 American Institute of Physics.

As Published: http://dx.doi.org/10.1063/1.3272264

Publisher: American Institute of Physics

Persistent URL: http://hdl.handle.net/1721.1/66700

Version: Final published version: final published article, as it appeared in a journal, conference proceedings, or other formally published context

Terms of Use: Article is made available in accordance with the publisher's policy and may be subject to US copyright law. Please refer to the publisher's site for terms of use. 


\section{A spin on cavity formation during water entry of hydrophobic and hydrophilic spheres}

Tadd T. Truscott and Alexandra H. Techet

Citation: Phys. Fluids 21, 121703 (2009); doi: 10.1063/1.3272264

View online: http://dx.doi.org/10.1063/1.3272264

View Table of Contents: http://pof.aip.org/resource/1/PHFLE6/v21/i12

Published by the American Institute of Physics.

\section{Related Articles}

On the dynamics and breakup of a bubble rising in a turbulent flow Phys. Fluids 23, 103301 (2011)

Visualization of flow patterns past various objects in two-dimensional flow using soap film Phys. Fluids 23, 091104 (2011)

Quantitative analysis of the dripping and jetting regimes in co-flowing capillary jets Phys. Fluids 23, 094111 (2011)

Microbubble formation and pinch-off scaling exponent in flow-focusing devices

Phys. Fluids 23, 092001 (2011)

Droplet formation in microfluidic cross-junctions

Phys. Fluids 23, 082101 (2011)

\section{Additional information on Phys. Fluids}

Journal Homepage: http://pof.aip.org/

Journal Information: http://pof.aip.org/about/about_the_journal

Top downloads: http://pof.aip.org/features/most_downloaded

Information for Authors: http://pof.aip.org/authors

\section{ADVERTISEMENT}

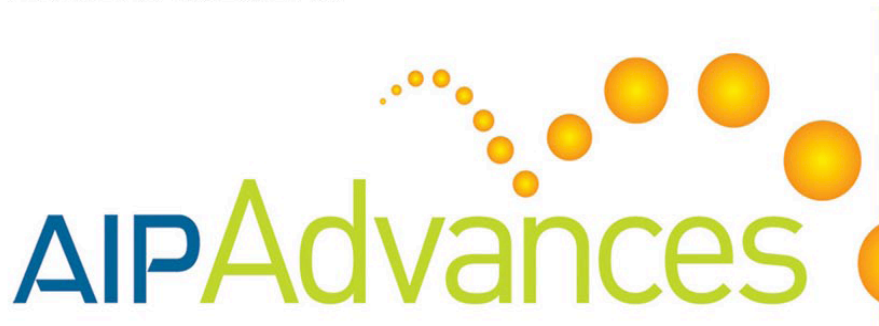

Submit Now

Explore AIP's new

open-access journal

Article-level metrics now available

Join the conversation!

Rate \& comment on articles 


\title{
A spin on cavity formation during water entry of hydrophobic and hydrophilic spheres
}

\author{
Tadd T. Truscott ${ }^{\mathrm{a})}$ and Alexandra $\mathrm{H}$. Techet ${ }^{\mathrm{b})}$ \\ Department of Mechanical Engineering, Massachusetts Institute of Technology, \\ Cambridge, Massachusetts 02139, USA
}

(Received 7 August 2009; accepted 13 November 2009; published online 31 December 2009)

\begin{abstract}
Surface coating and impact velocity can dramatically affect cavity formation during water entry of spheres. Duez et al. [Nat. Phys. 3, 180 (2007)] present a theoretical limit, dependent on impact velocity and surface static wetting angle, below which air cavities no longer form. We show that transverse spin alters the spheres surface velocity distribution to straddle this theoretical limit, resulting in cavity formation over half of the sphere and none on the other half, and yields similar results to the case of a sphere dropped without spin, at the same impact speed, when its surface is half hydrophilic and half hydrophobic. () 2009 American Institute of Physics.
\end{abstract}

[doi:10.1063/1.3272264]

The water entry of spheres presents a rich landscape over which to study the physics of the effect of surface treatment and kinematics on cavity formation and collapse. The classic images of water entry by spheres reveal a subsurface cavity that grows much larger than the sphere's volume and subsequently collapses, or pinches off, at some point below the free surface. ${ }^{1-3}$ The depth of pinch off is driven by the balance between inertia and hydrostatic forces, and is thus dependent on both impact Froude number $F r=U / \sqrt{g D}$ and mass ratio $m^{*}=\rho_{\text {sphere }} / \rho_{\text {fluid. }}{ }^{4,5}$

The condition of the sphere surface, e.g., static wetting angle $\theta_{0}$, can greatly affect the physics of water entry. Worthington $^{1}$ observed that when an already wetted (i.e., hydrophilic) sphere was dropped into a pool of water no underwater air cavity was formed and when the spheres were coated with soot (i.e., hydrophobic) the spheres made distinct air cavities. Korobkin and Pukhnachov ${ }^{6}$ detail the thin film of fluid that moves up and around the sphere in the early stages after impact; progression of this film around the sphere is greatly affected by surface treatment. ${ }^{7}$ Figure 1 illustrates the fundamental differences between the impact of hydrophilic spheres $\left(\theta_{0}<90^{\circ}\right)$ and hydrophobic spheres $\left(\theta_{0}>90^{\circ}\right)$ without spin for identical geometric and kinematic parameters. In the hydrophilic case, a simple vertical jet ejects from the free surface after impact but a subsurface air cavity does not form. Whereas in the hydrophobic case, a classic splash crown and subsurface air cavity form; the air cavity grows and subsequently collapses at pinch off. A model by Duez et al. ${ }^{7}$ offers a theoretical limit below which air cavities no longer form that is dependent on both impact velocity and static wetting angle.

The ultimate finding presented herein reveals the dramatic similarities between the effect of surface conditions, specifically static wetting angle, and the effect of spin on the formation of both the water entry cavity and the wedge. We demonstrate the ability to produce a fluid wedge that bisects

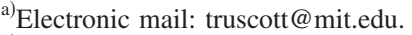

${ }^{b)}$ Electronic mail: ahtechet@mit.edu.
}

the cavity without using spin, by dropping a half-and-half sphere that has a hydrophobic surface treatment on one hemisphere and a hydrophilic treatment on the other. We suggest that the relative surface velocities on opposite sides of a spinning sphere straddle the demarcation line proposed by Duez et al. ${ }^{7}$ causing asymmetric cavity formation similar to a nonspinning half-and-half sphere. We contend that the spinning motion sets up a dynamic by which the wetting angle at the surface is altered; on the right side an upward, receding contact line decreases the wetting angle, thereby keeping the fluid in contact with the sphere, whereas on the left side an advancing contact line increases the wetting angle acting to encourage air entrainment and transferring greater energy into the formation of the cavity. Thus it is possible to use spin to setup a dynamic condition by which a sphere is effectively hydrophobic on one side (left) and hydrophilic on the other side (right).

Water entry experiments were performed in a large acrylic tank using standard billiard balls (diameter $D=2 R$ $=0.0572 \mathrm{~m}$ and density of $\rho=1740 \mathrm{~kg} / \mathrm{m}^{3}$ ). A custom apparatus was constructed that could both spin the spheres and drop them from a given distance above the free surface. High speed video captures the water entry event from two synchronized vantage points: top and side. The top images $(820 \times 920$ pixels $)$ are taken from directly above the sphere, and the lower images $(560 \times 820$ pixels $)$ are taken from the side. All images were acquired at 800 frames per second. Two types of surface treatments were used to create controlled static wetting angles $\theta_{0}$. Hydrophobic surfaces $\left(\theta_{0}=120^{\circ} \pm 10^{\circ}\right)$ were produced by spraying a thin coating of the chemical treatment WX2100, produced by Cytonix Corporation. Hydrophilic surfaces $\left(\theta_{0}=68^{\circ} \pm 10^{\circ}\right)$ were achieved by cleaning the spheres with acetone, isopropyl alcohol, and then ethanol, before allowing them to dry thoroughly. The roughness to diameter ratio of the hydrophobic coating was $k / d=1.96 \times 10^{-5}$, whereas the uncoated spheres had values of $k / d=0.80 \times 10^{-5}$.

Truscott and Techet ${ }^{4}$ first investigated the effect of spin on cavity formation by hydrophobic spheres, showing that 


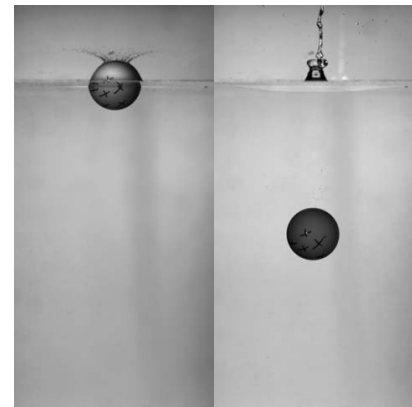

(a)

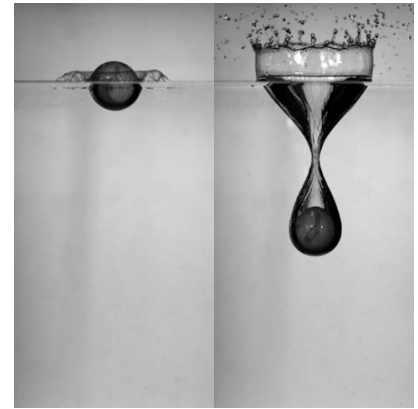

(b)
FIG. 1. Comparison of the water entry of two nonspinning billiard balls (diameter $=0.0572 \mathrm{~m}$ ) with the same impact speed $U_{0}=1.72 \mathrm{~m} / \mathrm{s}$. Case I is hydrophilic $\left(\theta=68^{\circ}\right)$ and does not form a cavity. Case II is hydrophobic $\left(\theta=120^{\circ}\right)$ and forms a splash crown and large subsurface air cavity. Images in both cases are separated by $97.5 \mathrm{~ms}$.

spin induces a lift force that acts to curve the sphere's trajectory and causes the cavity to form asymmetrically. The ratio of rotational velocity to downward velocity is given by the spin parameter $S=\omega R / U$, where $\omega$ is the angular velocity, $R$ is the sphere radius, and $U$ is the translational velocity of the sphere. Rotational velocities greater than $30 \%$ of the impact velocity $(S>0.3)$ result in pronounced asymmetric cavity cross sections due to the formation of a fluid wedge that is drawn up and around the sphere in the direction of rotation. ${ }^{4}$ Only small asymmetries are seen at lower $S$. Viscous effects ultimately lead to the decay of spin velocity, but not before the wedge of water fully bisects the cavity.

While a wide range of parameters have been studied, only five distinct, illustrative cases are detailed herein. The five cases were chosen to have the identical vertical surface impact velocity $U_{0}=1.72 \pm 0.1 \mathrm{~m} / \mathrm{s} \quad(F r=2.27)$ and alter only in surface treatment and spin rates. Cases I and II (Fig. 1) illustrate the effect of hydrophobic and hydrophilic spheres, respectively, with evenly coated surface treatments and no rotational velocity. Cases I and II are similar to the results shown by many previous studies and are given to illustrate the effect of chosen sphere surface treatments and for general comparison with cases III-V. Cases III and IV illustrate the effect of spin on a hydrophobic and a hydrophilic sphere, respectively; the spheres in these cases spin at an impact spin rate of $\omega_{0}=218$ and $192 \mathrm{rad} / \mathrm{s}(S=3.6$ and 3.2 ), respectively, in a counterclockwise direction (positive rotation by right hand rule) when viewed from the side, and from right to left in the top views. Case V is a nonspinning case, with a special half-and-half surface treatment applied; the left hemisphere is coated with the hydrophobic $\left(\theta_{\text {left }}=120^{\circ}\right)$ surface coating and the right hemisphere is cleaned such that it is hydrophilic $\left(\theta_{\text {right }}=68^{\circ}\right)$. Image sequences of the half-and-half sphere are captured at the same time instances as cases III and IV.

To better understand the differences between the five cases it is useful to relate the maximum and minimum vertical velocities on the sphere surfaces with the theoretical cavity prediction from Duez et al. ${ }^{7}$ The parameters for each case are superimposed on the theoretical plot from Duez et al. ${ }^{7}$ in Fig. 2, along with several additional spinning and nonspin-

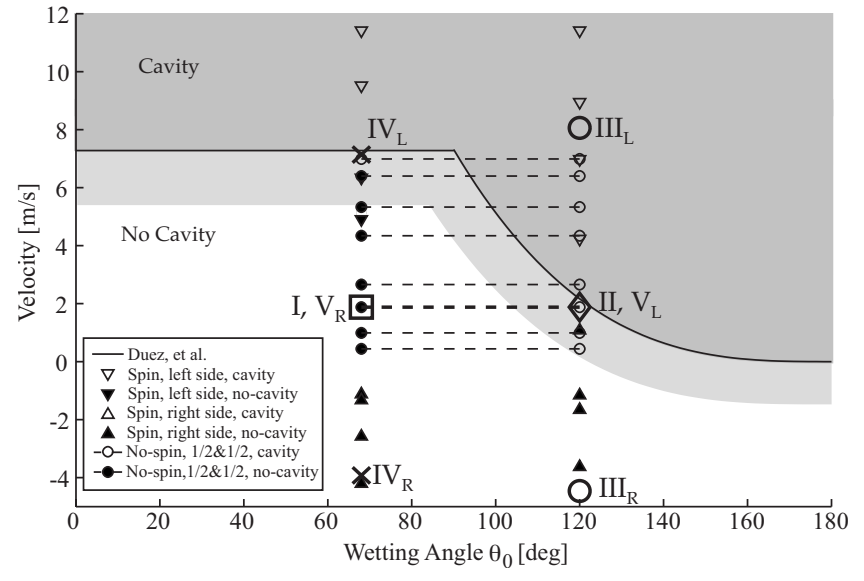

FIG. 2. Likelihood of cavity formation as a function of impact velocity $\left(U_{0}\right)$ and static wetting angle $\left(\theta_{0}\right)$. Case III ( $\bigcirc$ hydrophobic) and Case IV ( $X$ hydrophilic) have a rotational velocity that spans a vertical range for constant $\theta_{0}$, the left side has a faster relative velocity $\left(\mathrm{III}_{L}, \mathrm{IV}_{L}\right)$ than the right $\left(\mathrm{III}_{L}, \mathrm{IV}_{L}\right)$. All cases impact at the same velocity corresponding with cases I $(\square)$ and II $(\diamond)$, as well as case V, which is half hydrophilic $\left(V_{R}\right)$ and half hydrophobic $\left(V_{L}\right)$. The solid line is the theory proposed by Duez et al. (Ref. 7), above the line a cavity is formed (dark gray), below no cavity; the light gray band represents the region where cavity formation seems most sensitive to roughness and cavity formation occurs in some cases (filled markers). Additional half-and-half cases are plotted with smaller open circles, indicating cavity formation, and filled circles, indicating no cavity is formed, connected by a dashed line. Additional spinning cases, with varying impact and rotational velocities, are marked with open and filled triangles, forming cavity and no cavity, respectively; upside down triangles mark the left side of the sphere and right-side-up triangles mark the right side.

ning half-and-half cases investigated. In the two nonspinning cases, the sphere has the same vertical velocity relative to the free-surface at all points on the sphere, whereas in the spinning cases, the relative surface velocity differs at all points on the sphere due to the contributions from spin velocity. Spheres in cases I $(\square)$ and II $(\diamond)$ have uniform vertical velocity $U_{0}$ and a uniform static wetting angle over the entire sphere surface. Case I falls clearly within the no cavity region as expected from Fig. 1, and case II is very close to the theoretical curve and generates a classic water entry cavity (Fig. 1). The hydrophobic spheres studied create cavities at slightly lower impact velocity than the theory from Duez et $a l^{7}$ predicts, presumably due to roughness effects. In the mixed region, denoted by the light gray band under dashed curve in Fig. 2, we find that cavities are typically formed for the spheres we investigated in this study. The effect of roughness warrants further investigation.

Case V is performed with a constant vertical surface velocity over the entire sphere but with two distinct wetting angles on each half (left and right). Based on the theory by Duez et al., ${ }^{7}$ the right half of the sphere $\left(\mathrm{V}_{R}\right)$ has the same parameters (e.g., vertical velocity and static wetting angle) as those in case I and should yield no cavity formation. The left side $\left(\mathrm{V}_{L}\right)$ of the half-and-half sphere has the same parameters as those in case II and thus should theoretically result in cavity formation.

Upon impact, for a sphere rotating in a counterclockwise fashion, impacting at $U_{0}$, it would appear that the right side of the sphere is retreating out of the water and the left side is advancing into the fluid, since the relative velocity on the left 
and right sides of the sphere are different due to the rotational velocity. Considering, as a simple example, only the velocity along the equator separating the front and back hemispheres, for a spin parameter equal to one $S=\omega_{0} R / U_{0}$ $=1$, the left most point on the sphere has a tangential equatorial velocity equal to twice the impact velocity $\left(U_{L}=U_{0}\right.$ $\left.+\omega_{0} R=2\right)$, whereas the right most point has zero velocity $\left(U_{R}=U_{0}-\omega_{0} R=0\right)$. For $S>1$, the left hand side of the sphere has a velocity greater than twice the impact speed, and the right hand side has an upward velocity, which is negative since gravity is taken positive in the downward direction. The vertical surface velocity extrema for each case III and IV, with $S=3.6$ and 3.2, respectively, are found at the left and right most points on the sphere. Superimposed on the theoretical curve in Fig. 2, the velocity extrema indicate, for both cases III and IV, that the left side should generate a cavity and the right side should not. Top- and side-view image sequences for cases III-V (Fig. 3) are used to evaluate our hypothesis. The two spinning cases (III and IV) reveal that fluid is drawn up on the retreating side of the sphere and around the sphere surface, remaining in contact with the sphere as if there were no slip. The fluid wedge is drawn primarily into the center of the cavity along the equator, where tangential velocities are the greatest, with less fluid being drawn into the cavity near the poles, where the rotation does not affect the relative surface velocity.

In the hydrophobic spinning case (case III, $S=3.6$ ), the initial splash crown (about $t=3.25 \mathrm{~ms}$, not shown) radiates outward symmetrically at first, but begins to curl up along the equator as the sphere rotates; next a fluid wedge begins to form $(t=10 \mathrm{~ms})$. At time step $22.5 \mathrm{~ms}$, the top view shows the wedge has almost completely traversed the cavity and is affecting the splash asymmetry drawing a V-shaped, wedgelike section of the splash into the center of the cavity. The side view at $72.5 \mathrm{~ms}$ shows the air cavity covering the left most hemisphere. In the final time step $(97.5 \mathrm{~ms})$ a pronounced cardioid shape is seen in the top view, with the wedge fully bisecting the cavity. The velocity on the leftmost point on the sphere is $U_{L}=4.6 U_{0}=7.9 \mathrm{~m} / \mathrm{s}$ whereas the velocity on the right is $U_{R}=-2.6 U_{0}=4.47 \mathrm{~m} / \mathrm{s}$.

In the hydrophilic spinning case IV $(S=3.2)$ the initial splash crown appears even more asymmetric initially than in case III; the splash on the entire right half of the sphere appears to move up and around the sphere, whereas splash around the remainder of the sphere emanates radially outward in a classic fashion. Given only the vertical impact speed and the static wetting angle of case IV, theory would predict that this case should not form a subsurface air cavity over any portion of the sphere. However, a subsurface cavity is formed on the left hand side due the high relative velocity between the sphere and fluid $\left(U_{L}=4.2 U_{0}=7.2 \mathrm{~m} / \mathrm{s}\right)$, while the right side remains wetted due to the upward velocity $\left(U_{R}=-2.2 U_{0}=3.78 \mathrm{~m} / \mathrm{s}\right)$ and low static wetting angle, which both act to inhibit air entrainment and cavity formation. A wedge of fluid is also formed in this case bisecting the cavity, but it does not form the same distinct cardioid shape as in case III. For spinning, hydrophilic spheres with both relative surface velocities $U_{L}, U_{R}$ and impact velocity

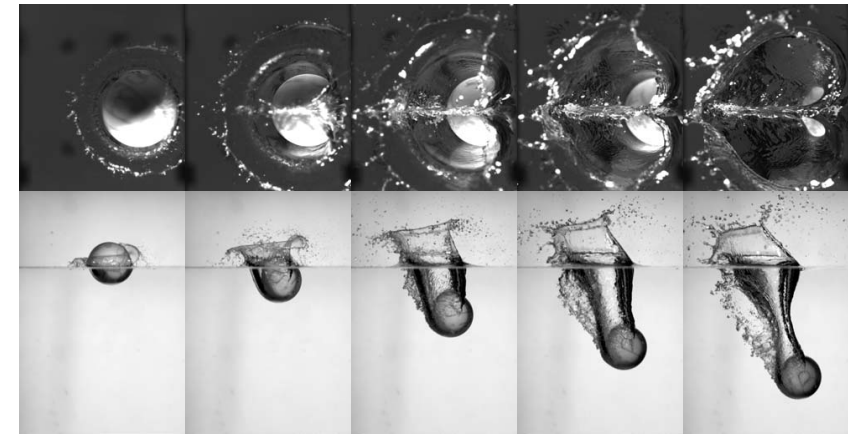

(a)

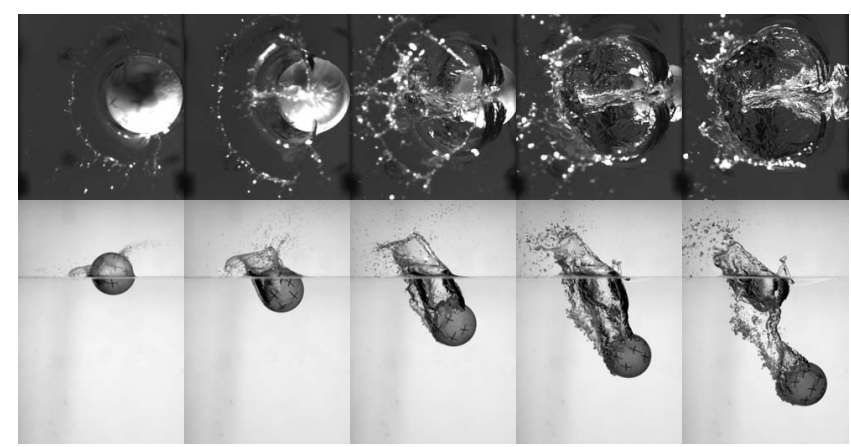

(b)

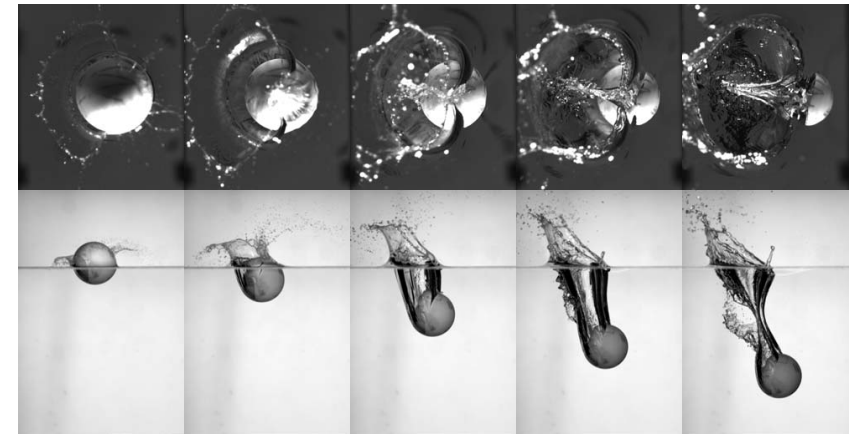

(c)

FIG. 3. Top and side views of the water entry of several billiard balls all with the same impact velocity $\left(U_{0}=1.72 \mathrm{~m} / \mathrm{s}\right)$. Case number, surface treatment and rotational velocity are indicated above each series of images. The spheres create a cardiod shaped splash curtain and curved subsurface air cavity as they descend in the fluid for the spinning hydrophobic, spinning hydrophilic, and nonspinning half-and-half cases.

$U_{0}$ below the theoretical line, we find no distinct cavity is formed.

The most interesting part of this study is that the dynamic effects of spin which cause wedge and asymmetric cavity formation can be duplicated quasistatically without spin, by simply dropping a sphere that is half hydrophilic and half hydrophobic. Case V (Fig. 3) shows a half hydrophobic (left half), half hydrophilic (right half) sphere entering the water with the same impact velocity as cases I-IV $\left(U_{0}=1.72 \mathrm{~m} / \mathrm{s}\right)$. It is not immediately obvious upon first glance that case III is much different than case IV. As the sphere impacts the free surface, water is drawn up the hydrophilic, right side, similar to case I, creating a fluid wedge similar to cases III and IV, but no outward cavity growth on the right. In case $\mathrm{V}$, air is entrained along the hydrophobic, left side, creating an air cavity and outward splash, as in case 


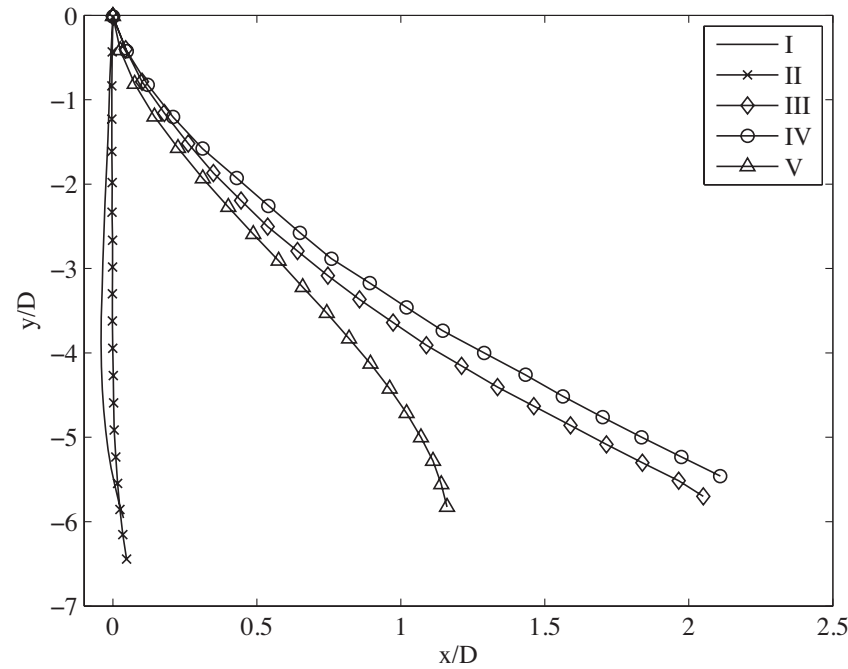

FIG. 4. Trajectory of the sphere in cases I-V (every tenth data point plotted).

II. The side view for case $\mathrm{V}$ reveals a distinct contact line along the equator between the two coated hemispheres.

Figure 2 hints at the possibility of asymmetric cavity growth but does not necessarily predict the wedge formation. The wedge formed in the half-and-half case is not as pronounced as in case III, but is highly similar to the wedge formed in case IV. Additional half-and-half cases tested (varying $U_{0}$ ) revealed similar trends to case $\mathrm{V}$ across a wide range of impact speeds from $U_{0}=0.5$ to $6.0 \mathrm{~m} / \mathrm{s}$ (Fig. 2). Experiments also showed that it was possible to have a cavity form on both sides if the impact speed was high enough $\left(U_{0}>7.0 \mathrm{~m} / \mathrm{s}\right)$. However, we could not create a case where no cavity was formed for a half-and-half sphere, likely due to the nature of the hydrophobic coating.

Despite the lack of spin in the half-and-half case, the sphere starts off on a curved trajectory (Fig. 4). The unbalanced momentum associated with the asymmetric cavity formation on the left side of the sphere forces the sphere to move to the right, similar to the spinning cases. The halfand-half coating does not impart any spin to the sphere, and thus once the cavity stops growing, the sphere in case V continues to move downward under the pull of gravity, since there is no spin induced lift. Initially cases III, IV, and V all exhibit similar levels of curvature in their trajectories. This suggests that the initial trajectory curvature for the spinning cases (III and IV) is dominated by asymmetric cavity formation, caused by the mismatch in relative velocities on either side of the spinning sphere, and not due to spin induced lift forces. Spin alters the boundary layer and wake structure generated by fully submerged spheres, and thus when the spheres are partially encased in the air cavity, these mechanisms are rendered ineffective. Once the air cavities collapse and the spheres are again submerged fully in water, spin induced lift takes over in the spinning cases. Eventually, viscous effects damp out the spin and the spheres fall to the bottom of the tank, ${ }^{4}$ as case $\mathrm{V}$ does after pinch off.

In conclusion, this study indicates that the dynamic effects of relative surface velocity can act similarly to static surface conditions. In the case of cavity forming spheres rotating in the transverse direction, a wedge of fluid is formed across the cavity from the side of least relative velocity to that with greatest relative velocity, effectively bisecting the cavity into two halves. The wedge formation and asymmetric cavity can be replicated for both hydrophobic and hydrophilic spheres when the critical relative velocity is exceeded on only one side of the sphere. Furthermore, these effects can be duplicated without rotating the spheres by coating the spheres half in a hydrophobic and half hydrophilic coating. This simple, yet elegant finding has applications to many naval hydrodynamics problems and should be taken into consideration when trajectory and/or cavity shape are important factors in engineering applications.

The authors gratefully acknowledge funding from the Office of Naval Research University Laboratory Initiative Grant No. N00014-06-1-0445.

${ }^{1}$ A. M. Worthington, A Study of Splashes (Longmans, Green, London, 1908).

${ }^{2}$ M. Lee, R. G. Longoria, and D. E. Wilson, "Cavity dynamics in highspeed water entry," Phys. Fluids 9, 540 (1997).

${ }^{3}$ V. Duclaux, F. Caillé, C. Duez, C. Ybert, L. Bocquet, and C. Clanet, "Dynamics of transient cavities," J. Fluid Mech. 591, 1 (2007).

${ }^{4}$ T. T. Truscott and A. H. Techet, "Water entry of spinning spheres," J. Fluid Mech. 625, 135 (2009).

${ }^{5}$ J. M. Aristoff and J. W. M. Bush, "Water entry of small hydrophobic spheres," J. Fluid Mech. 619, 45 (2009).

${ }^{6}$ A. A. Korobkin and V. V. Pukhnachov, "Initial stage of water impact," Annu. Rev. Fluid Mech. 20, 159 (1988).

${ }^{7}$ C. Duez, C. Ybert, C. Clanet, and L. Bocquet, "Making a splash with water repellency," Nat. Phys. 3, 180 (2007). 